\title{
A Two-Pheromone Trail Ant Colony System Approach for the Vehicle Routing Problem with Time Windows, Multiple Products and Product Incompatibility.
}

\author{
Andrés Palma, Industrial Engineer ${ }^{1}$, Daniela Cassandro, Industrial Engineer ${ }^{1}$, Stefanny Arboleda, Industrial Engineer, \\ and Carlos Paternina Arboleda, $\mathrm{PhD}^{1}$ \\ 1'Universidad del Norte, Colombia, acpalma@uninorte.edu.co, dcassandro@uninorte.edu.co, stefannya@uninorte.edu.co, \\ cpaterni@uninorte.edu.co
}

\begin{abstract}
This paper considers a variant of the Vehicle Routing Problem (VRP) subject to time windows for every customer, multiple products and incompatibility between them. Since the problem is NPhard, the approach to achieve feasible solutions is an Ant Colony System with Two-Pheromone with the objective of minimizing routing cost and vehicle fleet size. Experiments are carried out using instances from literature showing competitive solutions in relatively short computational times.

Keywords-- Vehicle routing, Time Windows, Multiple Products, Product Incompatibility, Ant Colony meta- heuristic.
\end{abstract}

\section{INTRODUCTION}

Flow of goods outside and within the supply chain is one of the most important activities in business logistics management since companies are not able to operate without the movement of raw material or finished products. In addition, is the most representative action in terms of costs for the greater number of organizations, considering that freight movement takes between one third and two thirds of total logistic costs [1]. This is why firms need to execute detailed studies on the transport of goods, in order to design a methodology that minimizes the costs produced by the transport and, in turn, decrease significantly the total supply chain costs.

This is how the Vehicle Routing Problem (VRP) seeks to minimize transport costs in the logistics supply chain by means of determining the optimal set of routes performed by a fleet of vehicles with determined capacity, considering time windows, drivers maximum working, and a large number of customers, each with a known demand level, which must be supplied from a single depot [2].

Dantzig and Ramser are considered the pioneers of this problem since they proposed the first mathematical and algorithmic formulation of the VRP applied in a delivery of gasoline to service stations real situation in 1959, and five years later, Clark and Wright improved the heuristic approach proposed by the first ones mentioned [3]. Supporting these two influential contributions and due to its potential applications in many real cases [4], a large amount of procedures have been proposed to approach VRP optimal solutions in different versions, applying optimization methods such as linear programming and branch and bound mostly used in smallmedium size problems, or heuristics and meta-heuristics that provide near-optimal solution for complex problems [4].

Digital Object Identifier (DOI): http://dx.doi.org/10.18687/LACCEI2018.1.1.293 ISBN: 978-0-9993443-1-6

ISSN: $2414-6390$
Ant Colony System (ACS) meta-heuristic is one of the most used algorithms since its number of successful applications reported in the Vehicle Routing Problem have demonstrated its effectiveness, as referred in [2]. With that knowledge in mind, in this paper is developed a two-pheromone trail ant colony system approach for a practical variant of the VRP in which a fleet of vehicles with limited transport capacity delivers multiple products to customers respecting time windows and incompatibility of products.

This paper continues with section 2 which reports information related to the studied area, section 3 describes the problem under study; section 4 presents the objective function to optimize; section 5 presents the two-pheromone trails ant colony system procedure proposed; experiments are shown in section 6, and finally some conclusions are presented in section 7.

\section{LITERATURE REVIEW}

The vehicle routing problem (VRP) can be described as the plan for designing an optimal delivery or ordering certain collection routes from one or more depots to many geographically dispersed cities or customers, subject to capacity restrictions, time, security, compatibility, among others. Considered as a combinatorial optimization problem with a computational complexity NP-hard [5], it is necessary to apply algorithms that will ensure the provision of a right solution in a reasonable computational time. In this way, different algorithms have been developed in order to achieve expected objectives, the implementation of exact algorithms in this problem based on linear programming or the branch and bound approach has been used for the solution of basic versions of the VRP as in [6], but considering the complexity of real world problems, metaheuristics have taken the lead due to its microcomputer capability and availability.

Solomon (1987), considered the design and analysis of algorithms for the routing of vehicles with time window constraints. Given the complexity of these types of problems, the author approaches methods of approach to study a set of problems, includes routing and programming environments that differ in terms of the type of data used to generate problems.

In [6] is proposed a Genetic Algorithm (GA) without trip delimiters, hybridized with a local search procedure, giving a flexible algorithm, simple and very effective to apply to two sets of standard benchmark instances ranging from 50 to 483

16 $^{\text {th }}$ LACCEI International Multi-Conference for Engineering, Education, and Technology: "Innovation in Education and Inclusion", 19-21 July 2018, Lima, Peru. 
customers. Tan et al. (2001) studied VRP applications with metaheuristics such as simulated annealing (SA), Taboo search (TS) and genetic algorithm (GA), other authors have studied too the implementation of these algorithms applying some modifications and strategies that improve the results in different types of VRP versions [2,8,10,11,12], and successfully implemented and deployed for real-life problems. Ant Colony System and powerful hybrids algorithms has been applied in many researches too, considering different types of restrictions, movements, strategies and modifications, demonstrating its effectiveness by the application of the methods in a considerable number of tests $[13,14,15,16]$.

Possible objectives may be to find a set of routes which minimizes the total distance travelled, or which minimizes the number of vehicles required and the total distance travelled with this number of vehicles. Baldacci et al. (2011) provided a review of the developments that had a great impact on the current cutting-edge algorithms for the vehicle routing problem (VRP). The authors review different mathematical formulations, relaxations and recent accurate methods for two of the most important variants of VRP: the trained VRP (CVRP) and the VRP with time windows (VRPTW). In their study they also facilitate a comparison of the computational performances of the different exact algorithms for the CVRP and VRPTW.

Another variants of the VRPs is the Vehicle Routing Problem with stochastic demands (VRPSD); in accordance with [5], in the literature have been two approaches for the solution of the VRPDS, The first is the static or a priori optimization and the second is the dynamic approach or optimization. Under the approach of a priori path, routes are established before knowing the demands of customers, so the vehicle distributes the goods following the route established client before starting the tour Since the demand for customers will only be known at the time for them, there may be a fault in the path, which means that the vehicle does not have the goods needed to satisfy the demands of the current node; for this, there are different strategies that avoid the modification of the sequence raised in an a priori.

\section{PROBLEM DESCRIPTION}

This paper focuses on a specific variant of the vehicle routing problem. This consists on defining routes for a homogeneous fleet of vehicles with limited capacity in order to supply clients with multiple types of products, which may not be compatible with each other. All the different types of product must be delivered within a predefined time window. The objective is to find a complete route for each vehicle that serves all the customers with a minimum cost (expressed in terms of travel distance or travel time). These routes must respect the time window for each customer, the vehicle capacity in terms of weight and volume, and the compatibility constraint associated with each type of product. Due to the compatibility constraint, each customer can be served by more than one vehicle depending on the type of demand.
An instance I of the Vehicle Routing Problem with Time Windows constraints and Multiple Products with Incompatibility constraints (VRPTWMPIC) consist on:

\section{A. Instance Description}

1) $G(V, E)$ : Is a directed graph where $\mathrm{V}$ is the set of $\mathrm{N}$ nodes representing the customers, with node 0 being the depot and $E$ is the set of edges connecting them.

2) D is a $N x N$ matrix that specifies travel distances.

3) $\mathrm{T}$ is a $N x N$ matrix that specifies travel times.

4) TW is a $N x 2$ matrix that specifies the time window for each customer. It contains the earliest and latest delivery times.

5) $\mathrm{S}$ is a $N$ matrix that specifies the service times for each customer.

6) $P$ is the number of product types for the demand.

7) $\mathrm{C}$ is a $P x P$ matrix that specifies the compatibility between each type of product.

8) $\mathrm{A}$ is a $N x P$ matrix that specifies the weight requested of product $\mathrm{p}$ for each customer.

9) $\mathrm{B}$ is a $N x P$ matrix that specifies the volume requested of product $\mathrm{p}$ for each customer.

10) $\mathrm{V}$ is a $M x 2$ matrix that specifies the capacity in terms of weight and volume for each vehicle.

\section{B. Objective Function}

This paper proposes an ant colony system approach for solving the VRPTWMPIC, defining as objectives the minimization of routing cost and vehicle fleet size. This approach seeks to solve the vehicle routing problem considering all the restrictions mentioned above. To determine the quality of a good solution, an objective function is defined as:

$$
F=\sum_{k=1}^{M} \xi_{k} * \sum_{j=1}^{N} X_{0 j k}+\sum_{i=0}^{N} \sum_{j=0}^{N} \sum_{k=1}^{M} C_{i j} * X_{i j k}
$$

Where $\mathrm{M}$ number of vehicles used, $\xi_{k}$ is fixed cost of vehicle $\mathrm{k}, \mathrm{N}$ is the number of nodes, $C_{i j}$ is the cost (represented as distance) of going from node $\mathrm{i}$ to $\mathrm{j}, X_{i j k}$ is a binary variable that takes the value of 1 if the vehicle $\mathrm{k}$ goes from node $\mathrm{i}$ to node $\mathrm{j}$ in the route, 0 otherwise.

The fixed cost $\xi_{k}$ is defined as:

$$
\xi_{k}=\sum_{0}^{N} \sum_{0}^{N} C_{i j}
$$

This objective function allows to control routing cost and fleet size, giving solutions that can be easily applied in a real situation, using less vehicles.

16 $^{\text {th }}$ LACCEI International Multi-Conference for Engineering, Education, and Technology: "Innovation in Education and Inclusion", 19-21 July 2018, Lima, Peru. 
IV. Two-Pheromone Trail ANT COLONy SySTEM APPROACH

The proposed approach uses a colony of cooperative agents to build a feasible solution for the VRPTWMPIC. It works as presented in figure 1.

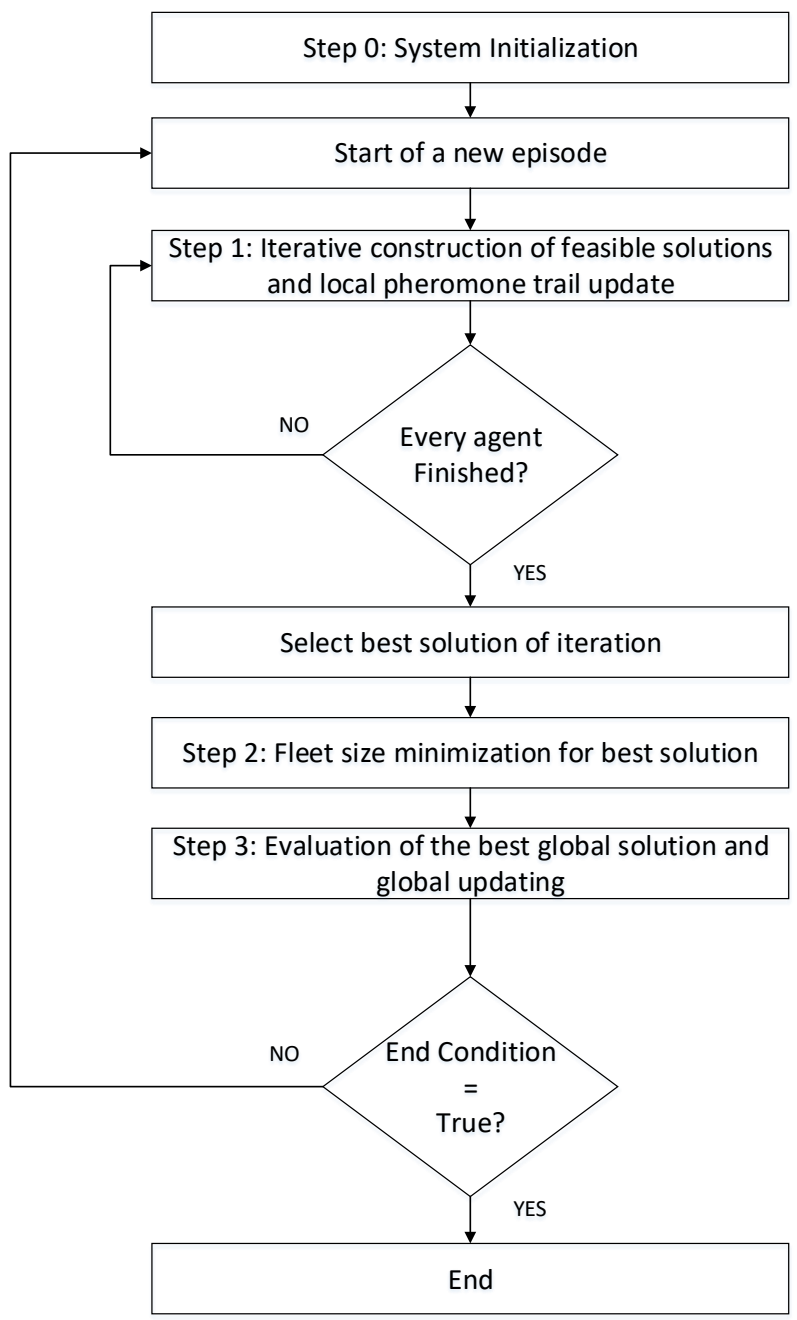

Figure 1. Two-pheromone trail ACS approach

A. Parameters

1) Number of iterations, $r \in Z^{+}$

2) Number of agents, $m \in Z^{+}$

3) Exploration rate, $w_{o} \in R, 0 \leq w_{o} \leq 1$

4) Learning rate, $\alpha \in R, 0 \leq \alpha \leq 1$

5) Discounting rate, $\gamma \in R, 0 \leq \gamma \leq 1$

6) Relative importance of pheromones, $\delta \in R, 0 \leq$ $\delta \leq 1$

7) Relative importance of heuristic, $\beta \in R, 0 \leq \beta \leq$ 1

8) Initial value of the pheromone $Q_{0} \in R, Q_{0} \geq 0$.

\section{B. Construction of Feasible Solutions}

The process to construct a feasible solution for every agent is shown in figure 2.

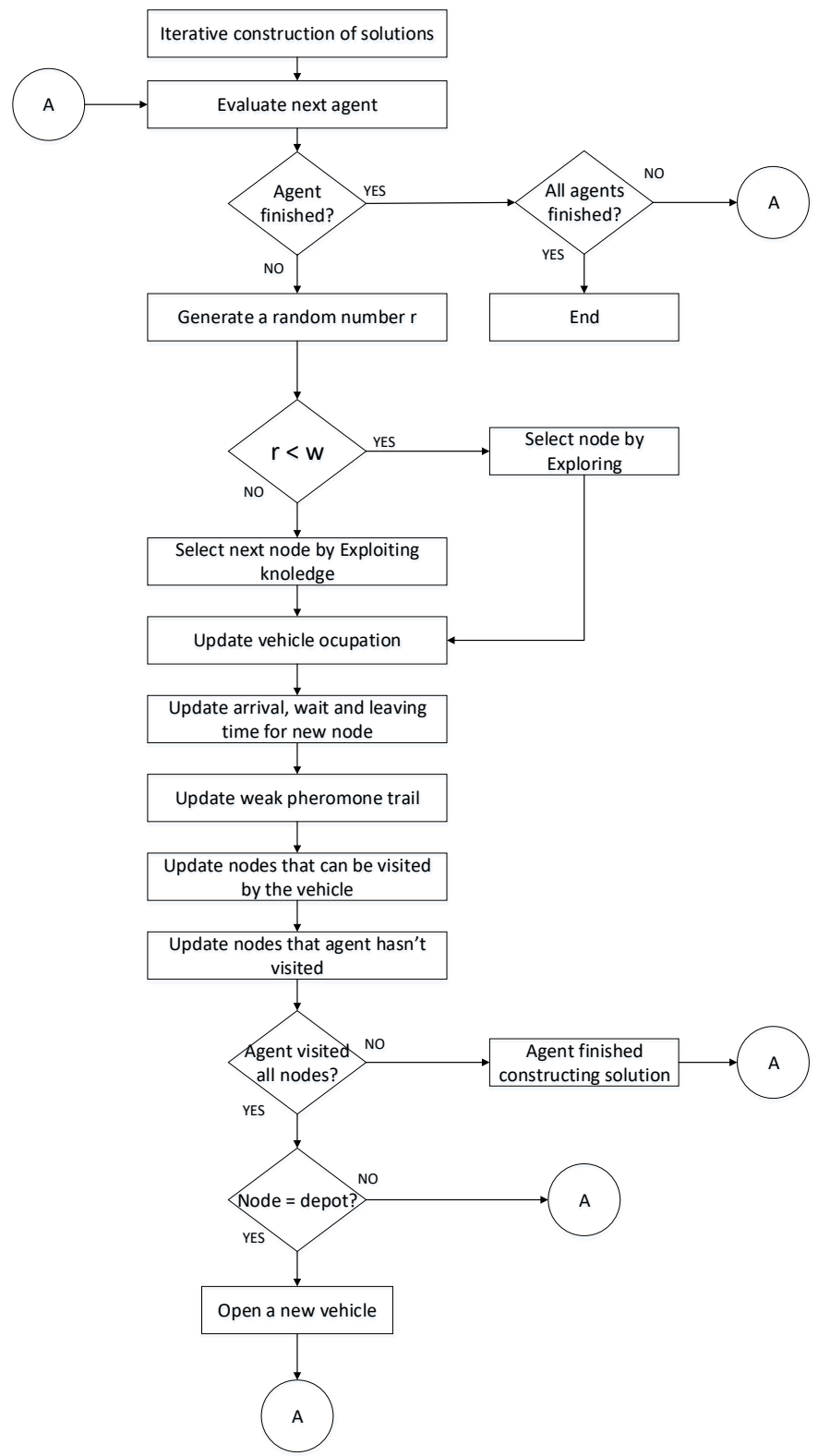

Figure 2. Iterative construction of feasible solutions.

When an agent decides to exploit the knowledge, it has to select a node based on the following formula:

$$
s_{a}=\arg \max \left\{\left|Q\left(r_{a}, s_{a}\right)\right|^{\delta}\left|H\left(r_{a}, s_{a}\right)\right|^{\beta}\right\} \quad s_{a} \in J_{a}\left(r_{a}\right)
$$

In the formula above, $Q\left(r_{a}, s_{a}\right)$ represents the amount of pheromones in the trail $\left(r_{a}, s_{a}\right)$, and $H\left(r_{a}, s_{a}\right)$ represents the desirability in short term to service a customer $s$, starting in $r$ for the agent a. This is expressed as:

16 $^{\text {th }}$ LACCEI International Multi-Conference for Engineering, Education, and Technology: "Innovation in Education and 


$$
\begin{aligned}
& H\left(r_{a}, s_{a}\right) \\
& =\frac{1}{\left(D\left(r_{a}, s_{a}\right) *\left(\max \left(v_{r}+\mathrm{T}\left(r_{a}, s_{a}\right), e_{s}\right)-v_{r}\right) *\left(l_{s}-v_{r}\right)\right)}
\end{aligned}
$$

With $D\left(r_{a}, s_{a}\right)$ being the distance between nodes $r_{a}$ and $s_{a}, v_{r}$ is the leaving time for the customer $\mathrm{r}, T\left(r_{a}, s_{a}\right)$ represents the time required to travel from customer $\mathrm{r}$ to $\mathrm{s}, e_{s}$ and $l_{s}$ corresponds to the earliest and latest delivery time for customer s (Time Window).

If the agent decides to explore, it selects randomly the next customer s according to the probability distribution:

$$
\operatorname{Pr}\left(r_{a}, s_{a}\right)=\frac{\left|Q\left(r_{a}, s_{a}\right)\right|^{\delta}\left|H\left(r_{a}, s_{a}\right)\right|^{\beta}}{\sum\left|Q\left(r_{a}, u\right)\right|^{\delta}\left|H\left(r_{a}, u\right)\right|^{\beta}} s_{a}, u \in J_{a}\left(r_{a}\right)
$$

\section{Weak and Strong Pheromone Trails}

This approach works with two pheromone trails, a weak pheromone trail or local trail and a strong pheromone trail or global trail. The first is updated by every agent in the construction of a feasible solutions while the other one is only updated at the end of an iteration and can only be updated by the agent with the best solution found at that moment.

The updates on the pheromone trails follows the next formulas, for weak and strong respectively.

$$
\begin{aligned}
& Q^{v}\left(r_{a}, s_{a}\right)=Q^{v}\left(r_{a}, s_{a}\right)+\alpha *\left[\gamma Q_{o}-Q^{v}\left(r_{a}, s_{a}\right)\right] \\
& Q^{\Lambda}\left(r_{a}, s_{a}\right)=Q^{\Lambda}\left(r_{a}, s_{a}\right)+\alpha *\left[\Delta Q-Q^{\Lambda}\left(r_{a}, s_{a}\right)\right]
\end{aligned}
$$

Where $Q^{v}$ and $Q^{\Lambda}$ represent the weak and strong pheromone trails and $\Delta Q$ is a reinforcement applied to the pheromone trail estimated as the inverse of the best current solution.

\section{Minimizing Fleet Size}

As mentioned in section II this approach seeks to minimize the fleet size and the total distance travelled. Once every agent finished constructing feasible solutions, the code select the best solution based on the objective function and proceeds to reduce the fleet size by removing clients from the vehicle with less number of costumers and assigning them on the best vehicle and position in the route as shown in figure 3 .

This procedure improves the objective value of the best solution of each iteration, transmitting that knowledge to the global pheromone trail and leading agents of the next iteration into building better solutions.

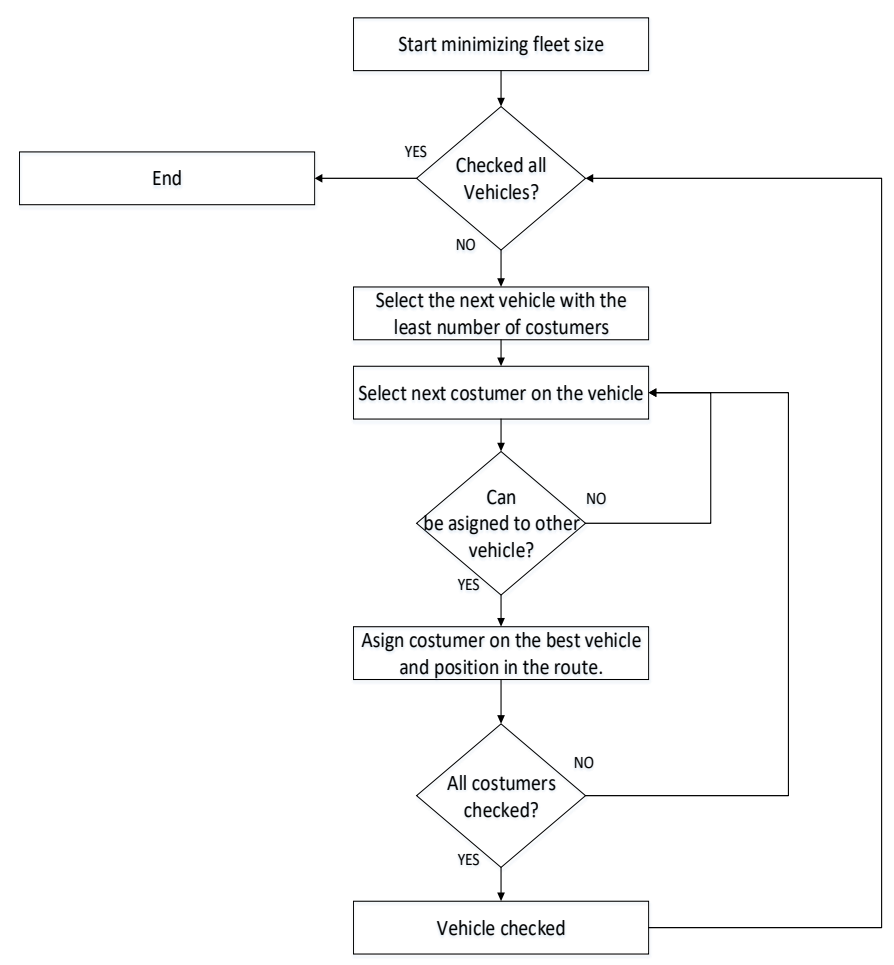

Figure 3. Minimizing fleet size.

\section{EXPERIMENTS}

\section{A. Parameter Setting}

The parameters were set as shown in the table 1 , as proposed by De la Cruz and Paternina (2013).

TABLE I

PARAMETERS OF THE ALGORITHM

\begin{tabular}{cc}
\hline Parameter & Value \\
\hline $\mathrm{r}$ & 75 \\
$\mathrm{~m}$ & 10 \\
$w_{o}$ & 0.05 \\
$\alpha$ & 0.1 \\
$\gamma$ & 0.3 \\
$\delta$ & 1 \\
$\beta$ & 1 \\
$Q o$ & $N / \sum_{0}^{N} \sum_{0}^{N} C_{i j}$ \\
\hline
\end{tabular}

\section{B. Test Instances}

The Solomon's repository (Solomon 2005) contains benchmark problems, with instances for the vehicle routing problem with time windows. Solomon's Instances Problems can be classified into three groups depending on the distribution of the customer locations: Random (R), clustered (C), and the mixture of both (RC). The VRP with Time Windows,

16 $^{\text {th }}$ LACCEI International Multi-Conference for Engineering, Education, and Technology: "Innovation in Education and 
Heterogeneous Fleet and Multiple Products was studied by De la Cruz and Paternina (2013). In that work, they adapted the instances R201 and R209 to the HVRPTWMP by adding a second type of demand (volume) and changing the capacity of the fleet to make it heterogeneous.

The proposed procedure is evaluated in four instances. First, the modified instances for the HVRPTWMP without compatibility constraints R201 and R209 as in De la Cruz and Paternina (2013) and Amador (2014). Then two adaptations of these previous instances to the VRPTWMPIC, considering 3 product types and incompatibility between them. The new instances are named R201_I and R209_I.

In the instances tested, travel costs between the customers are given in terms of the corresponding Euclidean distance and it is assumed that all vehicles perform a speed equals to a unit (having both the travel times and distances the same values). It also assumes that service time is always the same (product independent on load/unload). Also customer demands and vehicle capacities were redefined in terms of weight, volume and type denominations.

\section{Results and Computational Times}

Since the ant colony system approach is stochastic in nature, each instance was evaluated ten times, and the results are shown in tables 2, 3, 4 and 5. The best solution found for each instance are shown in the Annexes.

TABLE II

RESULTS FOR TEN RUNS OF INSTANCE R201

\begin{tabular}{cccc}
\hline R201 & Fleet & Distance & Error \\
\hline Best known solution & 4 & 1252,37 & $0 \%$ \\
Worst approach solution & 4 & 1648,83 & $32 \%$ \\
Best approach solution & 4 & 1524,85 & $22 \%$ \\
Average approach & & & \\
solution & 4 & 1610,16 & $29 \%$ \\
Standard Deviation & 0 & 39,17 & - \\
$95 \%$ CI Upper bound & 4 & 1634,44 & $31 \%$ \\
$95 \%$ CI Lower bound & 4 & 1585,88 & $27 \%$ \\
\hline
\end{tabular}

TABLE III

RESULTS FOR TEN RUNS OF INSTANCE R209

\begin{tabular}{cccc}
\hline R209 & Fleet & Distance & Error \\
\hline Best known solution & 3 & 909,86 & $0 \%$ \\
Worst approach solution & 3 & $1.540,26$ & $69 \%$ \\
Best approach solution & 3 & $1.242,06$ & $37 \%$ \\
Average approach & 3 & $1.440,36$ & $58 \%$ \\
solution & 0 & 101,76 & - \\
Standard Deviation & 3 & $1.503,43$ & $65 \%$ \\
$95 \%$ CI Upper bound & 3 & $1.377,28$ & $51 \%$ \\
$95 \%$ CI Lower bound & & & \\
\hline
\end{tabular}

TABLE IV

RESULTS FOR TEN RUNS OF INSTANCE R201_I

\begin{tabular}{ccc}
\hline R201_I & Fleet & Distance \\
\hline Worst approach solution & 10 & 3726,63 \\
Best approach solution & 9 & 3456,55 \\
Average approach & & \\
solution & 9,50 & 3565,07 \\
Standard Deviation & 0,53 & 93,06 \\
95\% CI Upper bound & 9,83 & 3622,75 \\
95\% CI Lower bound & 9,17 & 3507,39 \\
\hline
\end{tabular}

TABLE V

RESULTS FOR TEN RUNS OF INSTANCE R209_I

\begin{tabular}{ccc}
\hline R209_I & Fleet & Distance \\
\hline Worst approach solution & 8 & $3.138,89$ \\
Best approach solution & 7 & $2.964,25$ \\
Average approach & & \\
solution & 7,90 & $3.053,30$ \\
Standard Deviation & 0,32 & 65,29 \\
95\% CI Upper bound & 8,10 & 3093,76 \\
95\% CI Lower bound & 7,70 & 3012,83 \\
\hline
\end{tabular}

The solutions given by the procedure aren't close to the best solution found, however as seen in table 6, they are competitive with other heuristic approaches. Further research is required in order to improve the quality of the solution.

\begin{tabular}{lcc}
\multicolumn{4}{c}{ TABLE VI } \\
Comparative with OTHER HEURISTICS FOR INSTANCE R209 \\
\hline \multicolumn{1}{c}{ Heuristic } & Fleet & Distance \\
\hline \begin{tabular}{l}
\multicolumn{1}{c}{ Best known solution } \\
Two-pheromone trail ACS proposed
\end{tabular} & 3 & 909,16 \\
$\begin{array}{l}\text { 2-opt strategy (Osman and Christofides } \\
\text { 1989) }\end{array}$ & 4 & 1242,06 \\
$\begin{array}{l}\text { Simulated Annealing (Thangiah et al. } \\
\text { 1994) }\end{array}$ & 4 & 1206,56 \\
$\begin{array}{l}\text { Iterated Local Search (Thangiah et al. } \\
\text { 1994) }\end{array}$ & 4 & 1110,30 \\
$\begin{array}{l}\text { Location-based approach (Bramel and } \\
\text { Simchi-Levi 1995) }\end{array}$ & 3 & 1262,80 \\
$\begin{array}{l}\text { Tabu Search (Rochat and Taillard 1995) } \\
\text { Genetic Algorithms (Thangiah 1995) }\end{array}$ & 4 & 901,88 \\
$\begin{array}{l}\text { Probabilistic Tabu Search (Rochat and } \\
\text { Taillard 1995) }\end{array}$ & 3 & 1097,42 \\
$\begin{array}{l}\text { Constraint Programming (Shaw 1998) } \\
\text { MACS (Gambardella et al. 1999) }\end{array}$ & 3 & 944,64 \\
$\begin{array}{l}\text { Evolutionary Strategy (Homberger and } \\
\text { Gehring 1999) }\end{array}$ & 3 & 923,96 \\
\end{tabular}

It is to note that the incompatibility constraint generates big changes in the solutions found by the procedure in terms of distance and computational time (Table 7). This is expected

16 $^{\text {th }}$ LACCEI International Multi-Conference for Engineering, Education, and Technology: "Innovation in Education and 
because if a customer requests three types of products and they are not compatible between them, more than one vehicle is necessary to serve all the demand, generating more routing costs and incrementing the fleet size. This problem can be solved by considering using multi-compartment vehicles that allows to carry two incompatible products in the same vehicle, this approach is suggested for future research.

TABLE VII

COMPUTATIONAL TIMES FOR THE PROCEDURE (SECONDS)

\begin{tabular}{ccc}
\multicolumn{2}{c}{ COMPUTATIONAL TIMES FOR THE PROCEDURE (SECONDS) } \\
\hline & $\begin{array}{c}\text { Without } \\
\text { Incompatibility }\end{array}$ & $\begin{array}{c}\text { With } \\
\text { Incompatibility }\end{array}$ \\
\hline $\begin{array}{c}\text { Worst observed } \\
\text { time }\end{array}$ & 6 & 14,00 \\
$\begin{array}{c}\text { Best observed time } \\
\text { Average observed } \\
\text { time }\end{array}$ & 3 & 8,00 \\
$\begin{array}{c}\text { Standard Deviation } \\
\text { 95\% CI Upper } \\
\text { bound }\end{array}$ & 0,88 & 9,45 \\
$95 \%$ CI Lower & 4,23 & 1,32 \\
bound & 2,73 & 10,03 \\
\hline
\end{tabular}

\section{Conclusions ANd Future Research}

This paper considered a variant of the vehicle routing problem that has a lot of applications in real practices. This variant consist in the VRP subject to Time Windows, Multiple Products and Incompatibility between them. This paper proposes an approach to solve the VRPTWMPIC based on an ant colony optimization with a two-pheromone trail strategy, based on the procedure done by De la Cruz and Paternina (2013).

The procedure gives acceptable solutions in relatively short computational time, considering the amount of constraints and customers, however the algorithm can resolve any combination of the constraints enunciated above, e.g. Vehicle Routing Problem with Time Windows and Incompatible Products, resulting in a really practical and competitive procedure. The procedure is competitive in terms of capability of application in real life situations.

Since it is a one phased algorithm, the solutions can be improved by adding more phases to refine the feasible solution found, as shown by De la Cruz and Paternina (2013). Local Search procedures are suggested into further research.

It is expected that the computational times will increase with a larger problem size, meaning that it should be tested in future researches using instances with more than 100 customers, and quantify the effect in the computational time and quality of the solution.

\section{REFERENCES}

[1] R. Ballou, "Business logistics/supply management: planning, organizing, and controlling the supply chain" 5th. Ed., Pearson Education, Inc., 2004.

[2] B. Baker, \& M. Ayechew, "A genetic algorithm for the vehicle routing problem”, Computers \& Operations Research, vol. 30(5), pp. 787-800, 2003.
[3] P. Toth, \& D. Vigo, “The Vehicle Routing Problem", Society for Industrial and Applied Mathematics, 2002.

[4] J. De la Cruz, C. Paternina-Arboleda, V. Cantillo, \& J. Montoya-Torres, "A two-pheromone trail ant colony system - tabu search approach for the heterogeneous vehicle routing problem with time windows and multiple products". Journal of Heuristics, vol. 19(2), pp. 233-252, 2013.

[5] N. Gelves, R. Mora, \& H. Lamos, "Solving the vehicle routing problema with stochastic demands using spiral optimization, Universidad Industrial de Santader, Fac. Ing., vol. 25 (42), pp. 7-19. 2016.

[6] P. Toth, D. Vigo, "Models, relaxations and exact approaches for the capacitated vehicle routing problem", Discrete Applied Mathematics, vol. 123(1-3), pp. 487-512, 2002.

[7] K. Tan, L. Lee, Q. Zhu, K. Ou, "Heuristic methods for vehicle routing problema with time windows", Artificial Intelligence in Engineering, vol. 15(3), pp. 281-295, 2001.

[8] G. Laporte, M. Gendreau, J-Y. Potvin, F. Semet. "Classical and modern heuristics for the vehicle routing problem", International Transactions in Operational Research, vol. 7(4-5), pp. 285-300, 2000.

[9] F. Tang, \& R. Diéguez, "A tabu search algorithm for the vehicle routing problem with simultaneous pick-up and delivery service", Computers \& Operations Research, vol. 33(3), pp. 595-619, 2006.

[10] C. Prins, "A simple and effective evolutionary algorithm for the vehicle routing problem". Computers \& Operations Research, vol. 31(1), pp. 19852002, 2004.

[11] B. Kim, S. Kim, A. Sahoo, "Waste collection vehicle routing problem with time windows", Computers \& Operations Research, vol. 33(12), pp. 3624-3642, 2006.

[12] J. Dethloff, "Vehicle routing and reverse logistics: the vehicle routing problema with simultaneous delivery and pick-up", OR Spectrum, vol. 23, pp. 79-96, 2001.

[13] B. Bullnheimer, R. Hartl, C. Strauss, "An improved ant system algorithm for the vehicle routing problem", Annals of Operations Research, vol. 89(0), pp. 319-328, 1999.

[14] Y. Bin, Y. Zhong-Zhen, Y. Baozhen, "An improved ant colony optimization for vehicle routing problem", European Journal of Operational Research, vol. 196(1), pp. 171-176, 2009.

[15] J. Bell, P. McMullen, "Ant colony optimization techniques for the vehicle routing problem", Advanced Engineering Informatics, vol. 18(1), pp. 41-48, 2004.

[16] A. Donati, R. Montemanni, N. Casagrande, A. Rizzoli, L. Gambardella, "Time dependent vehicle routing problema with a multi ant colony system", European Journal of Operational Research, vol. 185(3), pp. 1174-1191, 2008.

[17] J. Renaud, G. Laporte, F. Boctor, "A tabu search heuristic for the multidepot vehicle routing problem", Computers \& Operations Researc, vol. 23(3), pp. 229-235, 1996.

[18] P. Shaw, "Using constraint programming and local search methods to solve vehicle routing problems", Principle and Practice of Constraint Programming, pp. 417-431, 1998.

[19] G. Laporte, H. Mercure, \& Y. Nobert. "An exact algorithm for the asymmetrical capacitated vehicle routing problem", Networks, vol. 16(1), pp. 33-46, 1986.

[20] G. Laporte, M. Desrochers, \& Y. Nobert. "Two exact algorithms for the distance-constrained vehicle routing problem", Networks, vol. 14(1), pp. 161$172,1984$.

[21] J. Homberger, H. Gehring, "Two evolutionary metaheuristics for the Vehicle Routing Problem with Time Windows" Information Systems and Operational Research, vol. 37(3), pp. 297-318, 1999.

[22] J. Amador-Fontalvo, C. Paternina-Arboleda, \& J. Montoya-Torres, "Solving the heterogeneous vehicle routing problem with time windows and multiple products via a bacterial meta-heuristic". International Journal of Advanced Operations Management, vol. 6(1), pp. 81-100, 2014.

[23] R. Baldacci, A. Mingozzi, R. Roberti, "Recent exact algorithms for solving the vehicle routing problem under capacity and time window constraints", European Journal of Operational Research, vol. 218(1), pp. 1-6, 2012.

[24] N. Christofides, \& S. Eilon, "An Algorithm for the Vehicle-Dispatching Problem", Journal of the Operational Research Society, vol. 20(3), pp. 309$318,1969$.

16 $^{\text {th }}$ LACCEI International Multi-Conference for Engineering, Education, and Technology: "Innovation in Education and Inclusion", 19-21 July 2018, Lima, Peru. 